\title{
Low-frequency internal friction in silica glass
}

\author{
F. Travasso ${ }^{1,2}$, P. Amico ${ }^{1,2}$, L. Bosi ${ }^{1,2}$, F. Cottone $^{1,2}$, A. Dari ${ }^{1,2}$, L. Gammaitoni ${ }^{1,2}$, H. Vocca $^{1,2}$ and \\ F. MARChESONI ${ }^{2,3,4}$ \\ ${ }^{1}$ Dipartimento di Fisica, Università di Perugia - I-06123 Perugia, Italy \\ ${ }^{2}$ Istituto Nazionale di Fisica Nucleare, VIRGO Project Sezione di Perugia - I-06123 Perugia, Italy \\ ${ }^{3}$ Dipartimento di Fisica, Università di Camerino - I-62032 Camerino, Italy \\ ${ }^{4}$ Michigan Center for Theoretical Physics, The University of Michigan - Ann Arbor, MI 48109-1120, USA
}

received 14 May 2007; accepted in final form 9 October 2007

published online 31 October 2007

\author{
PACS 04.80.Nn - Gravitational wave detectors and experiments \\ PACS 61.43.Fs - Glasses \\ PACS 62.40.+i - Anelasticity, internal friction, stress relaxation, and mechanical resonances
}

\begin{abstract}
Precise low-frequency internal friction measurements on vitreous silica, taken over a wide temperature $(4 \mathrm{~K}<T<300 \mathrm{~K})$ and frequency range $(40 \mathrm{~Hz}<\nu<14 \mathrm{kHz})$, show remarkable similarities, but also suggestive differences with recent light scattering experiments. In the interval $30 \mathrm{~K}<T<110 \mathrm{~K}$, the exponent $\alpha$ of the power law relaxational spectrum turns out to be proportional to $T-T_{k}$, with a minimum at around $T_{k}=13 \mathrm{~K}$. This phenomenon is interpreted as a manifestation of thermal activation in an asymmetric double well system, the asymmetry being associated to quenched spatial disorder. For $T>160 \mathrm{~K}$ the loss angle develops a distinct step-like structure followed by a plateau, both independent of $\nu$, thus signalling the onset of a competing relaxation mechanism with much higher an activation energy.
\end{abstract}

Copyright (c) EPLA, 2007

Introduction. - Interferometric detection of gravitational waves by means of Earth-based antennas, like the VIRGO detector [1], requires an extremely accurate characterization of all apparatus mechanical losses in order to achieve the target sensitivity of $10^{-21} \mathrm{~m} / \sqrt{\mathrm{Hz}}$ at operating frequencies below $100 \mathrm{~Hz}$. As part of this effort, we set up an experimental facility to measure energy losses due to both the internal friction of the optics components (mirrors, beam splitters, etc.) and the residual dissipation due to their mechanical suspensions (blade springs, fibers, etc.). Internal friction loss angles as small as $10^{-6}$ rad have been measured over broad temperature and frequency ranges for both crystalline and amorphous materials, like sapphire and fused quartz $[2,3]$.

In preparation for the upcoming second generation of the VIRGO detector, we resumed the study of the relaxation mechanisms in silica glasses, initiated by the Bayreuth group $[4,5]$, by performing internal friction measurements at frequencies ten times lower [6], and with resolution appreciably higher than reported in the earlier literature $[6,7]$.

Wide-spectrum relaxations are a signature of the glass state. They are responsible for broad quasi-elastic components in the neutron and light-scattering spectra $[8,9]$, in the attenuation of sound waves $[10-12]$, and in dielectric loss spectra [13]. The internal friction spectrum of vitreous silica, i.e., its loss angle measured at different frequencies [14], confirms quantitatively the existence of a broad distribution of relaxation rates $[4,10]$. For temperatures above $10 \mathrm{~K}$ such relaxations are modelled as thermal activation processes occurring in an appropriate distribution of asymmetric double-well potentials (ADWP) [10,15]; at lower temperatures, relaxation in an ADWP is expected to occur via quantum tunnelling for $T \lesssim 1 \mathrm{~K}$ [16], or dissipative quantum tunnelling for $1 \mathrm{~K} \lesssim T<10 \mathrm{~K}[17]$. Experimental data from internal friction [4,10,11], but also from sound wave attenuation $[18,19]$, allow direct access to the distribution of both the barrier heights, $g_{V}(V)$, and the asymmetry tilts, $g_{\Delta}(\Delta)$, of the ADWP.

Experimental setup. - We performed our measurements on $v-\mathrm{SiO}_{2}$ samples with relatively low resonance frequencies $\nu$ and $T$ varying from $4 \mathrm{~K}$ up to room temperature. Our internal friction data are consistent with the light-scattering results reported in refs. [4,5], but with some unexpected differences, namely: 1) The relaxational contribution to the loss angle is also a power law, $\phi \propto \nu^{\alpha}$ (fig. 1), but, at odds with ref. [4], $\alpha$ extrapolates to 0 for $T_{k}=13 \mathrm{~K}$ (fig. 2). Such a temperature off-set $T_{k}$ is by no means negligible and calls for a refinement of the ADWP 


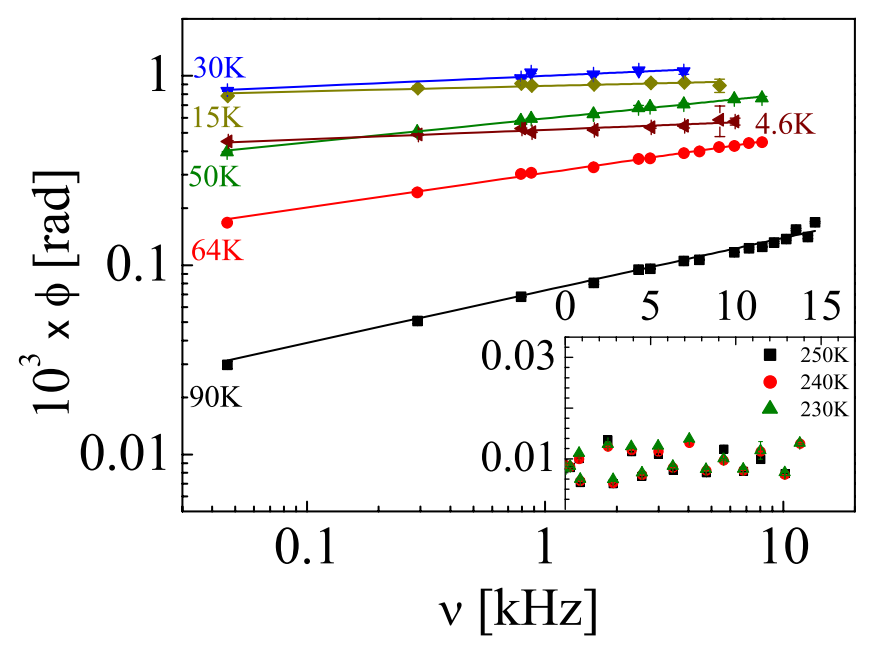

Fig. 1: (Color online) Internal friction angle $\phi v s . \nu$ at different temperatures: main panel, $T<110 \mathrm{~K}$; inset, $T>220 \mathrm{~K}$. The low- $T$ data in the main panel are fitted by the law $\phi=A \nu^{\alpha}$, the relevant exponents $\alpha(T)$ being plotted in fig. 2(a). The $T$-dependence of $A$ is analyzed in fig. 2(b), see also eq. (5). Error bars are drawn when exceeding the data-point thickness.

model. 2) for $T \approx 160 \mathrm{~K}, \alpha$ drops to zero and, correspondingly, the curves $\phi(T)$ exhibit an appreciable dip followed by a broad rebound bump at around $220 \mathrm{~K}$; at even higher temperatures, the loss angle finally grows almost insensitive, within our statistics, to both $T$ and $\nu$ (figs. 3 and 4 ). This observation suggests that at temperatures as low as half its average activation temperature $T_{0}=287 \mathrm{~K}$, the ADWP relaxation must be superseded by some structural relaxation mechanism, involving much higher activation energies.

A sample of Suprasil 311 (synthetic silica, Heraeus) of $(5 \times 45 \times 0.104) \mathrm{mm}^{3}$ was clamped at an angle with a copper clamp and placed in the vacuum chamber of a commercial cryostat at $10^{-5}$ mbar. The lowest 18 modes of the sample, in the $40 \mathrm{~Hz}<\nu<14 \mathrm{kHz}$ range, were excited electrostatically. The characteristic ring-down time $\tau_{\phi}$ of each resonant mode was determined by taking the Hilbert transform of the sample free vibration signal; the ensuing loss angle was computed as [14] $Q^{-1} \equiv \phi=\left(\pi \tau_{\phi} \nu\right)^{-1}$, where $Q$ is the quality factor of the corresponding mode.

The sample vibration signal was picked up by means of an optical lever. A Ne/He laser beam, shone on the sample, was reflected by its surface onto a quadrant side-by-side photodiode; the photodiode output signal was filtered by a pre-amplifier band-pass filter, in order to separate each mode, and acquired through an ADC system. Using standard low temperature control techniques, $\phi(T)$ for the first 18 modes was measured by ramping $T$ from $300 \mathrm{~K}$ down to $4 \mathrm{~K}$, with steps of $10 \mathrm{~K}$ taken at least 10 hours apart to avoid thermal stress effects. Validation checks ruled out appreciable effects due thermal hysteresis (by ramping the temperature up and down at different rates), spurious clamp losses (by changing clamp geometry and materials), and sample preparation (by cleaning and

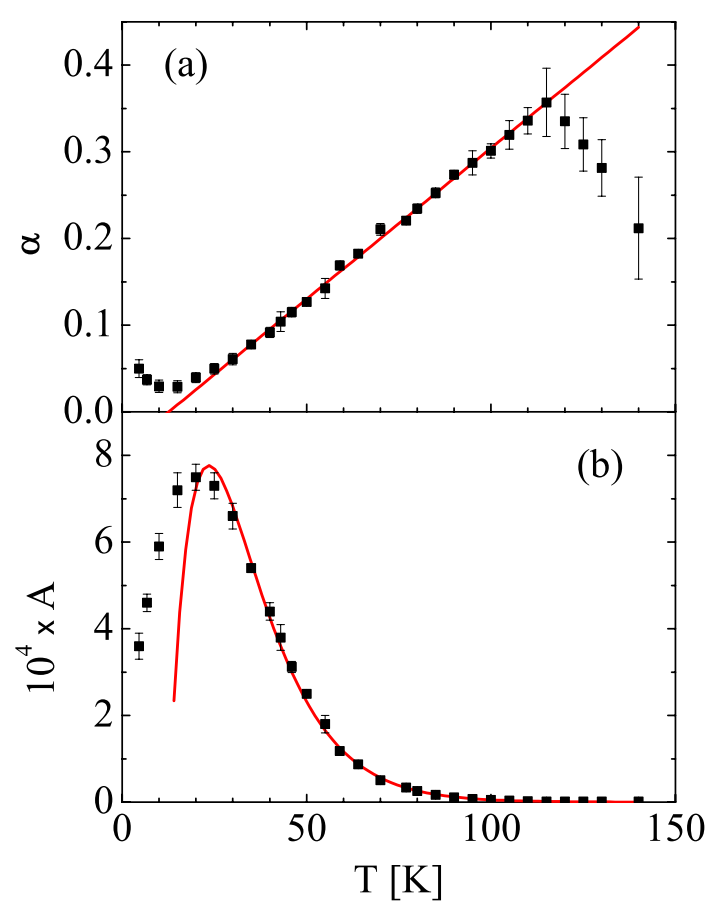

Fig. 2: (Color online) ADWP model interpretation: a) the data for $\alpha(T)$ from fig. 1(a) are fitted in the range $40 \mathrm{~K}<T<$ $110 \mathrm{~K}$ by the law (1) with $T_{0}=287 \pm 3 \mathrm{~K}$ and $T_{k}=12.6 \pm 0.6 \mathrm{~K}$; (b) the amplitude $A$ is plotted vs. $T$ and fitted by the ADWP law (5) with $C=0.055 \pm 0.005, \tau_{0}=0.5 \pm 0.2 \mathrm{ps} ; T_{0}$ and $T_{k}$ are as in (a).

annealing repeatedly the samples). The error on our $\phi$ data points was estimated to be, at worst, of the order of $0.5 \times 10^{-6} \mathrm{rad}$. As a general rule, when such an accuracy could not be achieved the relevant data points were not reported. This is the case, for instance, of the highest resonant modes in fig. 1: at low $T$, the ring-down time may get smaller than the vibration period, $\tau_{\phi} \nu \lesssim 1$, thus introducing systematic errors.

Low- $T$ results. - The intrinsic loss angle of $v-\mathrm{SiO}_{2}$ was thus determined as a function of both frequency (fig. 1) and temperature (fig. 3). The $\nu$-dependence of the internal friction is markedly different at low, $T<110 \mathrm{~K}$, and high temperatures, $T>160 \mathrm{~K}$. In fig. 1, main panel, the power law dependence $\phi \propto \nu^{\alpha}$ is apparent. The exponent $\alpha$ is independent of $\nu$ throughout the accessible frequency range, and increases linearly with $T$, even if the overall $\phi(\nu)$ magnitude gets progressively suppressed above $40 \mathrm{~K}$.

In fig. 2(a) we plotted the exponent $\alpha$ vs. $T$ at low temperature (see fits in fig. 1). Unlikely in ref. [4], for the interval $30 \mathrm{~K}<T<110 \mathrm{~K}$, our $\alpha(T)$ points were best fitted by the phenomenological law

$$
\alpha=\left(T-T_{k}\right) / T_{0} .
$$

While $T_{0}=287 \pm 3 \mathrm{~K}$ comes only $10 \%$ lower than the estimate obtained from light-scattering data [4], the 


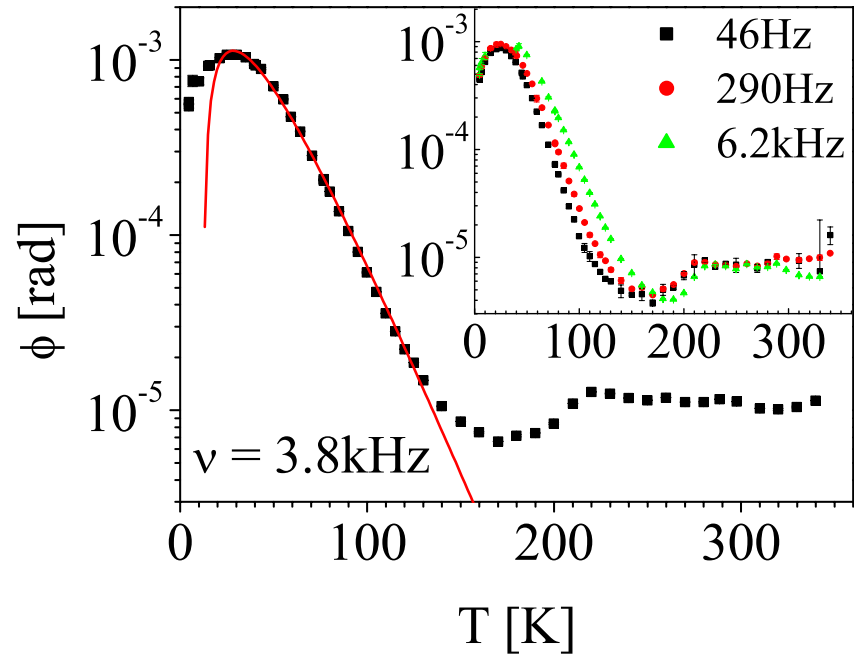

Fig. 3: (Color online) Internal friction loss angle $\phi v s . T$ for $\nu=3.8 \mathrm{kHz}$ (main panel) and different $\nu$, as indicated in the inset, for a comparison. The solid curve in the main panel denotes the ADWP contribution (5), obtained by combining the fits in figs. 1 and 2. Law (10) with $C_{g}=1.1 \times 10^{-5} \mathrm{rad}$, $T_{0}^{(g)}=1450 \mathrm{~K}$ and $\Delta_{0}^{(g)}=195 \mathrm{~K}$, is plotted as a dashed curve; the dashed line in the inset is our best estimate for the high- $T$ plateau (see text).

appearance of the off-set $T_{k}=12.6 \pm 0.6 \mathrm{~K}$ is a new finding that may have far reaching theoretical implications, as discussed below. (Earlier $\alpha(T)$ fits of light-scattering data, possibly compatible with the existence of some off-set $T_{k}$, went unnoticed [20].) Of course, the upward bending of $\alpha(T)$ for $T<T_{k}$ could not be reproduced by the fitting law (1), thus indicating a different relaxational ADWP regime, an issue still under investigation. Law (1) also fails for $T \lesssim T_{0} / 2$, so that we never measured values of $\alpha$ larger than 0.35 . This is another important difference with light-scattering experiments on $a-\mathrm{SiO}_{2}$ [5], where $\alpha$ was observed to approach 1 at room temperature and retain that value up to above the glass transition temperature $T_{g}$.

In the inset of fig. 1 the loss angles at high temperatures get suppressed below $10^{-5} \mathrm{rad}$ and become less sensitive to $\nu$, corresponding to the drop of $\alpha$ in fig. 2(a). This behavior is confirmed by the curves $\phi(T)$ at constant $\nu$ displayed in fig. 3, inset. Each curve $\phi(T)$ shows a peak at $T_{\max } \propto-\ln ^{-1} \nu$, a clear-cut signature of thermal activation [12], also compatible with the power law $\phi \propto \nu^{\alpha}$ and the linear $T$ dependence of $\alpha$ in eq. (1). However, on further increasing $T$, all $\phi(T)$ pass through a minimum at around $T_{0} / 2$ and then level off to a plateau, slightly below the $10^{-5}$ rad mark, for $T>250 \mathrm{~K}$; moreover, a broad step centered at around $220 \mathrm{~K}$ is also noticeable. These features weakly depend on the frequency over the entire $\nu$ range we explored, and, most remarkably, were never detected by means of other experimental techniques.

Our low- $T$ results can be interpreted within the classical ADWP model only under the key condition that thermal relaxation times in the model double wells is of the Kauzmann's type [21], i.e.

$$
\tau(T)=\tau_{0} \exp \left[V / k_{B}\left(T-T_{k}\right)\right]
$$

with exponentially distributed activation barriers [4],

$$
g_{V}(V)=\left(1 / V_{0}\right) \exp \left(-V / V_{0}\right)
$$

We also assume a Gaussian distribution for the asymmetry parameter $\Delta$,

$$
g_{\Delta}(\Delta)=\left(2 \pi \Delta_{0}\right)^{-1 / 2} e^{-\Delta^{2} / 2 \Delta_{0}^{2}}
$$

with $\Delta_{0}$ sufficiently large to warrant the approximate factorization $[18,19] \phi(\nu, T)=\phi_{V}(\nu, T) \phi_{\Delta}(T)$, with $\omega=2 \pi \nu$,

$$
\phi_{V}(\nu, T)=A(T) \nu^{\alpha(T)}=C \alpha(T)\left(\omega \tau_{0}\right)^{\alpha(T)},
$$

and $\alpha(T)$ as in eq. (1) for $V_{0} / k_{B}=T_{0}$. The factor $\phi_{\Delta}(T)=\left(\Delta_{0} / 2 k_{B} T\right) \operatorname{Erf}\left(2 k_{B} T / \Delta_{0}\right)$, with $\operatorname{Erf}(x)$ denoting the standard error function, is independent of $\nu$.

As mentioned above, the phenomenological laws (1) and (2) are not meant to apply at exceedingly low temperatures, $T \lesssim T_{k}$; therefore, the curves $\phi(T)$ have been fitted by means of $\phi_{V}(\nu, T)$, eq. (5), in the range $40 \mathrm{~K}<$ $T<110 \mathrm{~K}$ and for the values of $T_{0}$ and $T_{k}$ previously extracted in fig. 2(a). For the prefactor $\tau_{0}$ of the relaxation time (2) we obtained $\tau_{0}=0.5 \pm 0.2 \mathrm{ps}$ (i.e., twice the estimate reported in ref. [4]) and for the amplitude $A(T)$ the bell-shaped curve plotted in fig. 2(b). As expected, the fitted curve $A(T)$ fails to interpolate the experimental data for $T \lesssim 30 \mathrm{~K}$, resulting markedly lower for $T<20 \mathrm{~K}$.

In our fitting procedure, the slowly varying factor $\phi_{\Delta}(T)$ was incorporated in the coefficient $C(T)$, because it could not be resolved to a sufficiently good degree of confidence. However, asymmetry still plays a crucial role in our interpretation scheme. By choosing the Arrhenius factor in eq. (2), we implicitly advocated the presence of a mechanism capable of increasing the effective activation barriers, namely,

$$
V \rightarrow \frac{V}{1-\left(T_{k} / T\right)} \simeq\left[1+\left(T_{k} / T\right)+\left(T_{k} / T\right)^{2} \ldots\right]
$$

Now, as the fitted temperature $T_{k}$ is relatively low, one might think that on decreasing $T$ quantum mechanical effects can gradually be brought into play. However, corrections to the standard Arrhenius law [17] due to the so-called dissipative quantum tunneling (i.e., at the crossover between thermal activation and quantum tunneling) would rather lower the effective barriers [22], thus making the relaxation times shorter, at odds with our observations. Therefore, the existence of competing quantum relaxation processes in the same ADWP cannot be invoked here to explain the temperature off-set of eq. (1).

An ingredient capable of slowing down relaxation in a bistable system is spatial disorder [17]. In the 
glassy state, the atom groups undergoing configuration switches, are additionally subjected to local distortions that may randomly vary from site to site, so that no two activation processes ever occur over (through) the same potential barrier; moreover, for the temperatures and the observation times relevant to our measurements, one can regard such a spatial disorder as time independent (quenched). There are several ways one can account for spatial disorder in thermally activated processes [23]. In the present case we can establish a natural connection between double-well asymmetry and quenched disorder. In refs. $[4,10,15]$ local disorder is only responsible for the random tilting the symmetric double wells (separated by an average distance $d$ and an activation barrier $V$ ) with an appropriate asymmetry energy $\Delta$ distribution; at variance with such a mean field-like description, we propose to perturb the same symmetric double wells by superposing a quenched disorder field $\zeta(x)$ with Gaussian statistics and autocorrelation function

$$
\left\langle\zeta(x) \zeta\left(x^{\prime}\right)\right\rangle=\left(\sigma_{\zeta}^{2} / \lambda\right) \exp \left(-\left|x-x^{\prime}\right| / \lambda\right)
$$

One notes immediately that, for autocorrelation lengths $\lambda \sim d$ and standard deviations $\sigma_{\zeta} \sim \Delta_{0}, \zeta(x)$ may well reproduce, in mean-field approximation (leading order), the random tilts $\Delta$ of the ADWP model. However, as shown in ref. [23], the relaxation rate in a disordered double well gets modified to a larger extent than predicted in the mean-field approximation, namely by the ADWP model. Following the approach of ref. [23], we averaged the escape time over a given potential barrier $V$ with respect to all its spatial deformations $\zeta(x)$; we finally obtained an Arrhenius-like escape time corresponding to an increased effective activation barrier

$$
V \rightarrow V\left[1+\left(\Delta_{0}^{2} / V_{0} T\right)+\left(\Delta_{0}^{2} / V_{0} T\right)^{2} \ldots\right] .
$$

Note that extrapolating this expansion to higher orders, one recovers Kauzmann's relaxation rate (2). In view of this interpretation of the model asymmetry, $\Delta_{0}$ must be related to the temperature off-set $T_{k}$, that is, on comparing expansions (6) and (8) for $V$,

$$
\Delta_{0} / V_{0}=\sqrt{T_{k} / T_{0}}
$$

For the $v-\mathrm{SiO}_{2}$ samples considered in our experiments, typically $\Delta_{0} / V_{0} \simeq 0.2$, in good agreement with earlier estimates from ultrasonic data $[18,19]$.

High- $T$ results. - As shown in fig. 4, stricter accuracy criteria are required for internal friction measurements in silica glasses at high $T$, where $\phi$ values as small as $10^{-6} \mathrm{rad}$ have been recorded. To this purpose instead of clamping the sample at a corner by means of a copper clamp, we welded it by a corner to a thick cylinder of the same material, thus minimizing spurious losses [2]. As a result, the clamping loss plateau in fig. 4 got noticeably suppressed with two important consequences: the ADWP

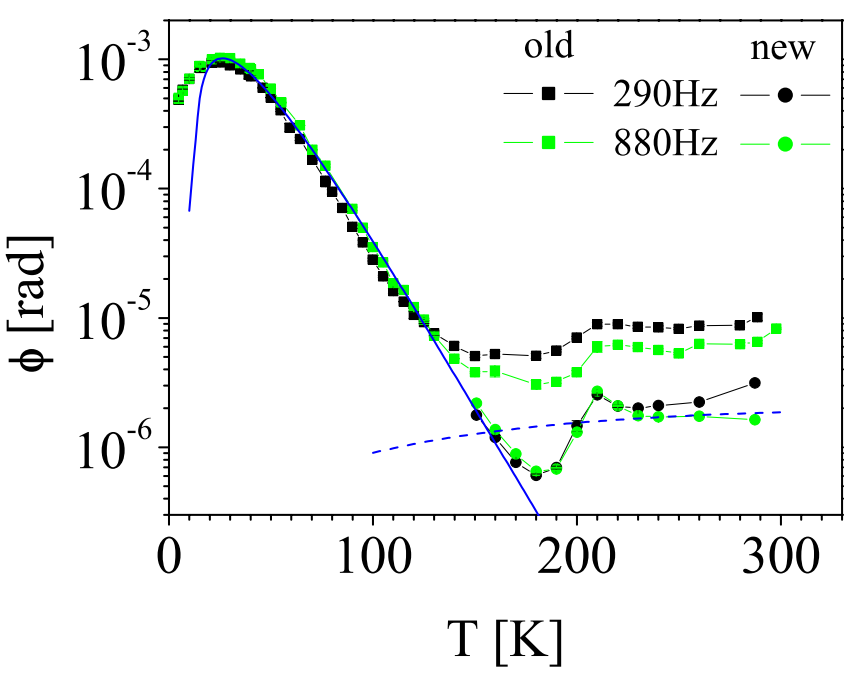

Fig. 4: (Color online) Internal friction loss angle $\phi$ vs. $T$ for two different sample holders: copper clamp, like in figs. 1-3 (squares) and welded sample (circles); data for two resonance frequencies are shown, $\nu=290 \mathrm{~Hz}$ (black dots) and $880 \mathrm{~Hz}$ (green dots). Theoretical fits refer to the new $\nu=290 \mathrm{~Hz}$ data set: the solid curve is the ADWP contribution (5) for the same fitting parameters as in fig. 3 ; the dashed curve is the law (10) for $C_{g}=0.3 \times 10^{-5} \mathrm{rad}, T_{0}^{(g)}=1450 \mathrm{~K}$ and $\Delta_{0}^{(g)}=195 \mathrm{~K}$.

law (4) could be extended up to $T \simeq 160 \mathrm{~K}$ with the same parameters extracted from the fits of fig. 3 , and the internal friction bump at around $T \sim 220 \mathrm{~K}$ could be established with higher experimental confidence.

Our high- $T$ internal friction data have relatively poorer statistics than at $T<110 \mathrm{~K}$; still, they clearly hint at the onset of a competing relaxation mechanism. In the ADWP model the monotonic function $\alpha(T)$ saturates to 1 for $T \gg T_{0}$ [5]. Nevertheless, the sudden drop of $\alpha(T)$ for $T \gtrsim T_{0} / 2$ (fig. 2(a)) does not mean that the assumptions of that model are incorrect. In fact, fig. 4, clearly shows that the predicted ADWP contribution (5) to $\phi$, solid curve, becomes negligible for $T \gtrsim 160 \mathrm{~K}$ (see also fig. 3, main panel). Above this temperature (and up to room temperature) internal friction is dominated by a distinct dissipation mechanism with $\alpha \sim 0$. Indeed, on inspecting the insets of figs. 1 and 3 (clamped sample) and fig. 4 (welded sample), it is apparent that at high- $T$ the curves $\phi(\nu)$ are substantially $\nu$-independent, although rather noisy (within the data error). Vice versa, the curves $\phi(T)$ recorded at constant $\nu$, being less noisy, show a neat crossover between the ADWP relaxation mechanism and a new one, in the form of a dip. Such a mechanism gets activated by mechanical deformations, like those we applied to our $v-\mathrm{SiO}_{2}$ samples, but not, for instance, by light scattering [4].

The contribution of such a relaxation mechanism to the internal friction angle can be separated into a broad steplike structure at $T \sim 220 \mathrm{~K}$, followed by a plateau for $T \lesssim$ $250 \mathrm{~K}$, both showing a weak frequency dependence, if any. 
Our best data, displayed fig. 4, support this conclusion, regardless of the experimental uncertainty.

The $\phi(T)$ plateau can be phenomenologically explained by assuming that at high- $T$ structural relaxations, like the buckling of the $\mathrm{SiO}_{2}$ groups in glassy chains [24,25], with large activation energies $V^{(g)}$, i.e., $V_{0}^{(g)} \gg V_{0}$, come into play. Formally, one can still employ the ADWP framework leading to eq. (5), but with two important differences: i) for $T \ll T_{0}^{(g)}$, here $T_{0}^{(g)} \equiv V_{0}^{(g)} / k_{B}$, the relevant exponent $\alpha_{g}$ is typically quite small. According to eq. (1), $\alpha_{g}(T) \propto T / T_{0}^{(g)}$ where $T_{0}^{(g)}$ is chosen to coincide with the glass transition temperature of $v-\mathrm{SiO}_{2}, T_{g} \simeq$ 1450 K. ii) Asymmetry corrections, if applicable, occur at energy scales not much larger than $\Delta_{0}$, so that $\Delta_{0}^{(g)} \ll$ $V_{0}^{(g)}$. As a result, the factor $\phi_{\Delta}(T)$ in eq. (5) can be approximated to $\Delta_{0}^{(g)} / 2 k_{B} T$ and, consequently, $\phi(\nu, T)$ attains the plateau

$$
\phi(\nu, T)=C_{g} \alpha_{g}(T) \phi_{\Delta}(T) \simeq C_{g} \Delta_{0}^{(g)} / 2 V_{0}^{(g)}
$$

with $V_{0}^{(g)}=k_{B} T_{g}$. In fig. 4 a frequency-independent plateau $\phi(T)=(2 \pm 0.5) \times 10^{-6} \mathrm{rad}$ is indeed observable for $T \lesssim 250 \mathrm{~K}$. Note that the ensuing estimate of $C_{g}$ is two orders of magnitude smaller than the constant $C$ extracted from the low-temperature data of fig. 2(b). This remark hints at the possibility of a detailed comparison between volume density, nature and coupling to the strain field of the activated relaxation groups in the two opposite temperature regimes.

Finally, the bump at $T \sim 220 \mathrm{~K}$ can be regarded as due to either the step-like branch of $\phi(T)$, predicted by the approximate law (10) and fitted in fig. 4, or to a weak resonance peak superposed to it, possibly related to the sample geometry [2]. The accuracy of our data, so far, is not good enough to discard any such interpretation.

Conclusions. - In conclusion, we have shown that internal friction experiments at low frequencies and low temperatures provide a fresh insight into the relaxation mechanisms of vitreous silica. At variance with other experimental techniques, like light-scattering [4], internal friction is characterized by the (ADWP) relaxation of glassy defects for temperatures up to about $120 \mathrm{~K}$, above which the precursors of structural glassy relaxation(s) set in $[5,24,25]$. Similarities and differences between light-scattering and internal friction observations at low frequency can only be explained by direct comparison of the two experimental techniques.

\section{REFERENCES}

[1] Caron B. et al., Class. Quantum Grav., 14 (1997) 1461.

[2] Амico P. et al., Rev. Sci. Instrum., 73 (2002) 179; 3318; Alshourbagy M. et al., Rev. Sci. Instrum., 77 (2006) 044502.

[3] Penn S. D. et al., Phys. Lett. A, 352 (2006) 3; Numata K. et al., Class. Quantum Grav., 19 (2002) 1697.

[4] Wiedersich J., Adichtchev S. V. and Rössler E., Phys. Rev. Lett., 84 (2000) 2718; Wiedersich J. et al., Phys. Rev. B, 64 (2001) 064207.

[5] Adichtchev S. V. et al., J. Non-Cryst. Solids, 307-310 (2002) 24.

[6] Raychaudhuri A. K. and Hunklinger S., Z. Phys. B, 57 (1984) 113.

[7] Fine M. E., van Duyne H. and Kenney N. T., J. Appl. Phys., 25 (1954) 402; CAhill D. G. and vaN Cleve J. E., Rev. Sci. Instrum., 60 (1989) 2706.

[8] Winterling G., Phys. Rev. B, 12 (1975) 2432.

[9] Buchenau U. et al., Phys. Rev. Lett., 60 (1988) 1318.

[10] Hunklinger S. and von Schickfus M., Amorphous Solids: Low-Temperature Properties, edited by PHILliPS W. A. (Springer-Verlag, Berlin) 1981, p. 81.

[11] Keil R., Kasper G. and Hunklinger S., J. Non-Cryst. Solids, 164-166 (1993) 1183; TopP K. A. and CAHILL D. G., Z. Phys. B, 101 (1996) 235; Surovtsev N. V. et al., Phys. Rev. B, $\mathbf{5 8}$ (1998) 14888.

[12] Tielbürger D. et al., Phys. Rev. B, 45 (1992) 2750.

[13] Fontella J. et al., J. Non-Cryst. Solids, 31 (1979) 401.

[14] Nowick A. S. and Berry B. S., Anelastic Relaxation in Crystalline Solids (Academic, New York) 1972.

[15] Theodorakopoulos N. and Jäckle J., Phys. Rev. B, 14 (1976) 2637; Gilroy K. S. and Phillips W. A., Philos. Mag. B, 43 (1981) 735; for a review, see Phillips W. A., Rep. Prog. Phys., 50 (1987) 1657.

[16] Anderson P. W., Halperin B. I. and Varma C. M., Philos. Mag. B, 25 (1972) 1.

[17] Hänggi P., Talkner P. and Borkovec M., Rev. Mod. Phys., 62 (1990) 251.

[18] Bonnet J. P., J. Non-Cryst. Solids, 127 (1991) 227.

[19] Vacher R., Courtens E. and Foret M., Phys. Rev. B, 72 (2005) 214205.

[20] Gainaru G. et al., Phys. Rev. B, 72 (2005) 174203.

[21] Kauzmann W., Chem. Rev., 43 (1948) 219.

[22] Grabert H., Weiss U. and Hänggi P., Phys. Rev. Lett., 52 (1984) 2193.

[23] Engel A. and Moss F., Phys. Rev. A, 38 (1988) 571; Marchesoni F., Europhys. Lett., 8 (1989) 83; Phys. Rev. $E, 56$ (1997) 2492.

[24] Nakayama T., Rep. Prog. Phys., 65 (2002) 1195.

[25] Granato A. V. and Khonik V. A., Phys. Rev. Lett., 93 (2004) 155502; NordLund K. et al., Europhys. Lett., 71 (2005) 625. 